\title{
Circadian Rhythms and the Treatment of Rheumatoid Arthritis
}

\author{
Yool Lee* \\ Department of Biomedical Sciences, Washington State University, USA
}

*Corresponding author: Yool Lee, Department of Biomedical Sciences, Elson S Floyd College of Medicine, Washington State University, Spokane, WA 99202, USA.
Received Date: April 03, 2021

Published Date: April 14, 2021

\begin{abstract}
Circadian clocks are ubiquitous biological timing systems that drive roughly 24 rhythms of our behavior (e.g., sleep/wake, feeding/fasting cycles) and physiology (e.g., metabolism, hormonal system, immune functions) through daily adjustments to environmental day and night cycles. Besides the rhythmic regulation of physiological processes, the circadian clockwork has been increasingly recognized to determine the daily variation in pathological symptoms and severity, particularly in autoimmune diseases including Rheumatoid Arthritis (RA). RA is a chronic inflammatory disease caused by the body's immune system attacking its own tissues and joints, resulting in major symptoms such as joint pain and stiffness. In most RA patients, the autoimmune symptoms are pronounced in the morning and are mediated by circadian rhythms of leukocyte recruitment to inflamed tissues, release of pro-inflammatory cytokines (e.g., IL-6, TNF- $\alpha$ ), and immune-modulatory hormone levels (e.g., glucocorticoid, melatonin). With the growing knowledge of the circadian etiology of RA clinical symptoms, here, we will briefly discuss recently established chronotherapeutic concepts as well as approaches to improve outcomes of RA treatment.
\end{abstract}

Keywords: Circadian clock; Rheumatoid arthritis; Autoimmune diseases; Glucocorticoid, Melatonin; Chronotherapeutic treatment

\section{Introduction}

Circadian clocks are cell-autonomous timing systems that generate roughly 24-hour periodic rhythms that are conserved in nearly all life from unicellular organisms to humans. These internal timing systems integrate with diverse environmental (e.g., light) and metabolic (e.g., food intake) stimuli to regulate biological activities, including sleep/wake, feeding times, energy metabolism, and hormonal and immune functions [1]. Disturbances of these rhythms caused by sleep deprivation, mistimed eating, or chronic jet lag are closely associated with the development of obesity, diabetes, and cancer, as well as allergic and immunological diseases [2-4]. Beyond the circadian regulation of physiology, clinical studies indicate that multiple diseases exhibit time of day variations in symptoms and severity [5]. In particular, autoimmune diseases, such as Rheumatoid Arthritis (RA), exhibit higher severity in the morning than at night in most patients [6,7]. According to animal and human model studies, hyper-morning inflammatory symptoms are associated with night (inactive phase) synthesis and the release of pro-inflammatory cytokines and chemokines, as well as the nocturnal rise of immune cell trafficking, proliferation, and phagocytosis at the site of inflammation [7]. Such chronic inflammatory conditions in RA severely compromise the nighttime availability of endogenous anti-inflammatory glucocorticoid, and, conversely, increase the pro-inflammatory melatonin hormone level, which contributes to disease severity [8]. Thus far, experimental and clinical evidence has shown that daily administration of exogenous glucocorticoids or anti-inflammatory drugs (e.g., NSAIDs, methotrexate) at bedtime is more effective than in the morning for the prevention and treatment of morning RA clinical symptoms $[7,9]$. In this short review, we will briefly discuss further details regarding the link between circadian rhythms and RA, highlighting treatment strategies that exploit chrono pathophysiologic mechanisms. 


\section{The Circadian Clock and the Immune System}

In all living organisms, the basic biological timing unit is the cell. In mammals, including humans, circadian rhythms are primarily driven by a cell-autonomous molecularfeedbackmechanismin which the circadian transcriptional activators aryl hydrocarbon receptor nuclear translocator-like (BMAL1) and circadian locomotor output cycles kaput (CLOCK) cyclically activate the expression of their own repressors Period (PER)/Cryptochrome (CRY) [10]. The core clock machinery is mobilized to reset cellular rhythms upon intrinsic and extrinsic stimuli, involving multiple post-translational (e.g., phosphorylation, sumoylation) and metabolic (e.g., Ca2+, cAMP) signaling pathways [11-14]. Systemically, multi-stimuli responsive oscillators in neurons and fibroblasts are tightly coupled to form the brain and peripheral organ clock systems, which, in turn, constitute a body-wide circadian network with adaptive synchronous capacity for environmental entrainment of rhythmic behaviors (e.g., sleep/ wake and feed/fast cycles) and physiology (e.g., temperature, blood pressure, metabolism, immune functions [1]. In particular, the hypothalamic suprachiasmatic nucleus (SCN), a central circadian pacemaker in the brain, communicates retinal light input to peripheral clock systems, thus mediating periodic synchronization of internal body rhythms with external day and night cycles. For example, the SCN coordinates the rhythmic peak secretion of melatonin sleep hormone in the pineal gland at night as well as glucocorticoid (cortisol in humans) stress hormone in the adrenal glands in the morning via sympathetic innervations, thus ensuring sleep/wake cycles [15]. Similarly, the SCN also conveys external timing information to the immune system through endocrine and autonomic pathways, such as local adrenergic innervation of $\beta 2$-adrenergic receptors ( $\beta 2 \mathrm{ARs}$ ) expressed on lymphocytes [16]. Interactions between the circadian and immune systems are bidirectional in that most immune cells contain the molecular clocks that drive the rhythmic synthesis and release of immune factors, which, in turn modulate the circadian phases of body clock systems [17,18]. Mirroring the circadian-immune connection, circadian disruptions, such as environmental desynchronization (e.g., sleep deprivation, jet lag, mistimed feeding) and/or molecular clock dysfunctions (e.g., host or immune cell-specific mutations of core clock genes), lead to the dysregulation of immune responses and inflammation that can further disrupt circadian rhythms $[2,19]$. Consistent with these findings, increasing numbers of epidemiological studies have reported that night shift workers, transatlantic air travelers, and people with sleep disorders are at greater risk of developing RA than controls [20-25].

\section{Circadian Rhythms in Rheumatoid Arthritis}

Accumulating evidence has shown that circadian clocks control the timing of symptoms and severity of chronic inflammatory diseases, including RA $[2,26]$. RA is a chronic, progressive inflammatory disease with massive infiltration of inflammatory cells into the synovium of multiple joints, causing major symptoms, such as pain, inflammation, and stiffness. It has been well- documented that RA symptoms exhibit a nearly 24-hour circadian rhythm, with maximum pain and stiffness in the early morning $[2,7]$. These symptoms are associated with increased release of pro-inflammatory cytokines (including TNF- $\alpha$ and IL-6) from hyper-activated immune cells, such as macrophages, at night and in the early morning, favoring edema and the accumulation of synovial fluid that results in morning stiffness and swelling [6]. In this regard, the daily anti-phasic fluctuation of circadian hormones, such as the lowest level of anti-inflammatory cortisol and the highest level of pro-inflammatory melatonin at night, contributes to the morning RA symptoms. The elevated melatonin level at night stimulates the secretion of pro-inflammatory cytokines, including IFN-g, IL-1, IL-6, and TNF- $\alpha$, from immune-modulatory cells [7]. This increase in cytokines promotes hepatic expression of $11 \beta$-hydroxysteroid dehydrogenase, which converts cortisol, the most potent endogenous anti-inflammatory hormone, into inactive cortisone [6]. This conversion results in abnormal hyposecretion of cortisol during the night, contributing to the presence of early morning clinical joint symptoms in patients with RA [7]. Notably, this vicious cycle appears to be further potentiated by a higher level and earlier increase (at least 2 hours earlier than its normal peak at $2 \mathrm{am}$ ) of melatonin in RA patients compared to control subjects, probably due, in part, to the altered chronophysiology caused by the chronic nature of RA $[17,27,28]$. Considering these findings, the use of melatonin intended to induce sleep is not recommended for people with RA or other inflammatory or autoimmune diseases [23].

\section{Circadian Treatment of Rheumatoid Arthritis}

Based on the circadian pathophysiology of RA symptoms, timed administration of an exogenous glucocorticoid has been widely implemented as the main treatment strategy for RA patients to replenish the insufficient availability of the endogenous antiinflammatory hormone at night $[2,7,9]$. Indeed, several clinical studies have shown that treatment with glucocorticoids late at night is more effective at relieving or preventing morning symptoms in RA patients because it inhibits nocturnal increases in the proinflammatory cytokines IL-6 and TNF- $\alpha[6,8]$. Earlier studies also showed that a dose of prednisolone, a synthetic glucocorticoid, at night (10pm-11 pm or $2 \mathrm{am})$ resulted in a significantly shorter duration of morning stiffness than the same dose provided in the morning (6 am-7 am) [29,30]. Furthermore, in subsequent CAPRA (Circadian Administration of Prednisone in RA) clinical studies, Modified-Release (MR) prednisone taken at night (administration at $10 \mathrm{pm}$ and release from the capsule after $4 \mathrm{~h}$ at 3-4 am) was shown to be highly effective in abolishing the IL-6 morning peak, with improvement in morning stiffness that was substantially greater than what was observed with conventional immediaterelease prednisolone [31-33]. Similar to the glucocorticoid chronotherapy results, Non-Steroidal Anti-Inflammatory Drugs (NSAIDs) taken at night have been reported to be more effective in controlling morning signs and symptoms of RA than either morning 
or daytime dosing. For example, initial double-blind crossover trials showed that flurbiprofen and indomethacin resulted in improved morning symptoms for the bedtime dose group [34,35]. Subsequent studies using various modes of controlled-release administration confirmed that several NSAIDs, such as ketoprofen [36], indomethacin [37], aceclofenac [38], and lornoxicam [39], were effective at relieving RA morning symptoms when taken at bedtime. Disease-Modifying Anti-Rheumatic Drugs (DMARDs), such as methotrexate, have also been used as alternative RA cure agents to benefit patients who experience RA disease progression and joint damage despite conventional treatment with steroids and NSAIDs. A previous study using arthritis animal models showed that methotrexate exhibited the most efficient anti-inflammatory effects when administered in phase with the 24 hours cycling of TNF- $\alpha$ [40]. A subsequent study involving Japanese RA patients confirmed that methotrexate administration three times weekly at nighttime was more effective at reducing TNF- $\alpha$ levels and RA symptoms compared to the conventional dosing protocol (a dose in the morning and afternoon on day 1 , and in the morning on day 2) [41]. In line with these results, recent studies with arthritis animal models showed that daily administration of low-dose methotrexate was more effective than weekly administration according to the IL-6 circadian rhythm in reducing arthritis score as well as TNF- $\alpha$, IL-6, and $\mathrm{C}$ reactive protein (CRP) levels [42,43]. Overall, these findings suggest that targeting circadian immune pathologic rhythms with anti-rheumatic hormones and drugs could improve treatment outcomes for those with RA.

\section{Conclusion}

In recent decades, extensive chronobiological studies have increased our understanding of the circadian nature of the physiology and pathology of immune systems. This has allowed for the establishment of mechanism-based chronotherapeutic interventions for autoimmune diseases, including RA. Along with the progressive development of manipulatable drug-release technologies, current anti-rheumatic treatment protocols with glucocorticoids, NSAIDs, and DMARDs at bedtime have proven to be more efficacious at improving morning RA symptoms, relative to daytime dosing schemes, via preemptive prevention of the nocturnal rise in pro-inflammatory immune activity (e.g., nightly surge of cytokines). Beyond RA therapies, circadian mechanisms of immunopathogenesis can be applied to the treatment of other inflammatory diseases. For example, a recent clinical trial study reported that dexamethasone (dex), a synthetic glucocorticoid, reduced the death rate of critically ill Covid-19 patients by about one-third, perhaps by suppressing hyperactive immune responses in the lung, such as acute respiratory distress syndrome (ARDS), caused by the viral infection [44]. Considering the success of treating chronic autoimmune diseases with steroid chronotherapy, it is reasonable to assume that nighttime glucocorticoid delivery would likely be helpful in reducing the severe immunopathological consequences (ex., cytokine storm) observed in patients with
Covid-19 infection. Moreover, in future clinical studies, it would be beneficial and important to take chronobiology into account for improving the safety and efficacy of existing or novel therapeutic interventions used for the management of RA and/or other immune-related diseases.

\section{Acknowledgement}

We would like to thank Amy Sullivan, Ph.D. from Obrizus Communications for assisting with the helpful editing of this article.

\section{Conflicts of Interest}

No conflict of interest.

\section{References}

1. Koronowski KB, Sassone Corsi P (2021) Communicating clocks shape circadian homeostasis. Science 12: 371(6530).

2. Scheiermann C, Gibbs J, Ince L, Loudon A (2018) Clocking in to immunity. Nat Rev Immunol 18(7): 423-437.

3. Lee Y, Lahens NF, Zhang S, Bedont J, Field JM, et al. (2019) G1/S cell cycle regulators mediate effects of circadian dysregulation on tumor growth and provide targets for timed anticancer treatment. PLoS biology 17(4): e3000228.

4. Panda S (2016) Circadian physiology of metabolism. Science 354(6315): 1008-1015.

5. Ruben MD, Smith DF, FitzGerald GA, Hogenesch JB (2019) Dosing time matters. Science 365(6453): 547-549.

6. Spies CM, Straub RH, Cutolo M, Buttgereit F (2014) Circadian rhythms in rheumatology-a glucocorticoid perspective. Arthritis research \& therapy 16 Suppl 2: S3.

7. Cutolo M (2019) Circadian rhythms and rheumatoid arthritis. Joint Bone Spine 86(3): 327-333.

8. Buttgereit F, Smolen JS, Coogan AN, Cajochen C (2015) Clocking in: chronobiology in rheumatoid arthritis. Nature reviews Rheumatology 11(6): 349-356.

9. Jacob H, Curtis AM, Kearney CJ (2020) Therapeutics on the clock: Circadian medicine in the treatment of chronic inflammatory diseases. Biochemical pharmacology 182: 114254.

10. Takahashi JS (2017) Transcriptional architecture of the mammalian circadian clock. Nature reviews Genetics 18(3): 164-179.

11. Lee Y, Lee J, Kwon I, Nakajima Y, Ohmiya Y, et al. (2010) Coactivation of the CLOCK-BMAL1 complex by CBP mediates resetting of the circadian clock. Journal of cell science 123(Pt 20): 3547-3557.

12. Lee Y, Chun SK, Kim K (2015) Sumoylation controls CLOCK-BMAL1mediated clock resetting via CBP recruitment in nuclear transcriptional foci. Biochimica et biophysica acta 1853(10 Pt A): 2697-2708.

13. Shim HS, Kim H, Lee J, Son GH, Cho S, et al. (2007) Rapid activation of CLOCK by $\mathrm{Ca} 2+$-dependent protein kinase $\mathrm{C}$ mediates resetting of the mammalian circadian clock. EMBO reports 8(4): 366-371.

14. Travnickova Bendova Z, Cermakian N, Reppert SM, Sassone Corsi P (2002) Bimodal regulation of mPeriod promoters by CREB-dependent signaling and CLOCK/BMAL1 activity. Proceedings of the National Academy of Sciences of the United States of America 99(11): 7728-7733.

15. Scheiermann C, Kunisaki Y, Frenette PS (2013) Circadian control of the immune system. Nat Rev Immunol 13(3): 190-198.

16. Suzuki K, Hayano Y, Nakai A, Furuta F, Noda M (2016) Adrenergic control of the adaptive immune response by diurnal lymphocyte recirculation through lymph nodes. The Journal of experimental medicine 213(12): 2567-2574. 
17. Poolman TM, Gibbs J, Walker AL, Dickson S, Farrell L, et al. (2019) Rheumatoid arthritis reprograms circadian output pathways. Arthritis research \& therapy 21(1): 47.

18. Keller M, Mazuch J, Abraham U, Eom GD, Herzog ED, et al. (2009) A circadian clock in macrophages controls inflammatory immune responses. Proceedings of the National Academy of Sciences of the United States of America 106(50): 21407-21412.

19. Castanon Cervantes O, Wu M, Ehlen JC, Paul K, Gamble KL, et al. (2010) Dysregulation of inflammatory responses by chronic circadian disruption. Journal of immunology 185(10): 5796-5805.

20. Coskun Benlidayi I (2018) Sleep impairment: an obstacle to achieve optimal quality of life in rheumatoid arthritis. Rheumatol Int 38(12): 2183-2192.

21. Chung WS, Lin CL (2018) Sleep disorders associated with risk of rheumatoid arthritis. Sleep Breath 22(4): 1083-1091.

22. Hsiao YH, Chen YT, Tseng CM, Wu LA, Lin WC, et al. (2015) Sleep disorders and increased risk of autoimmune diseases in individuals without sleep apnea. Sleep 38(4): 581-586.

23. Torres Ruiz J, Sulli A, Cutolo M, Shoenfeld Y (2017) Air Travel, Circadian Rhythms/Hormones, and Autoimmunity. Clin Rev Allergy Immunol 53(1): 117-125.

24. Hedstrom AK, Akerstedt T, Klareskog L, Alfredsson L (2017) Relationship between shift work and the onset of rheumatoid arthritis. RMD Open 3(2): e000475.

25. Irwin MR, Olmstead R, Carrillo C, Sadeghi N, Fitzgerald JD, et al. (2012) Sleep loss exacerbates fatigue, depression, and pain in rheumatoid arthritis. Sleep 35(4): 537-543.

26. Hand LE, Hopwood TW, Dickson SH, Walker AL, Loudon AS, et al. (2016) The circadian clock regulates inflammatory arthritis. FASEB J 30(11): 3759-3770.

27. Habers GEA, van der Helm van Mil AHM, Veldhuijzen DS, Allaart CF, Vreugdenhil E, et al. (2021) Earlier chronotype in patients with rheumatoid arthritis. Clinical rheumatology.

28. Cutolo M, Sulli A, Pizzorni C, Secchi ME, Soldano S, et al. (2006) Circadian rhythms: glucocorticoids and arthritis. Annals of the New York Academy of Sciences 1069: 289-299.

29. De Silva M, Binder A, Hazleman BL (1984) The timing of prednisolone dosage and its effect on morning stiffness in rheumatoid arthritis. Annals of the rheumatic diseases 43(6): 790-793.

30. Arvidson NG, Gudbjornsson B, Larsson A, Hallgren R (1997) The timing of glucocorticoid administration in rheumatoid arthritis. Annals of the rheumatic diseases 56(1): 27-31.

31. Buttgereit F, Doering G, Schaeffler A, Witte S, Sierakowski S, et al. (2008) Efficacy of modified-release versus standard prednisone to reduce duration of morning stiffness of the joints in rheumatoid arthritis (CAPRA-1): a double-blind, randomised controlled trial. Lancet 371(9608): 205-214.
32. Buttgereit F, Doering G, Schaeffler A, Witte S, Sierakowski S, et al. (2010) Targeting pathophysiological rhythms: prednisone chronotherapy shows sustained efficacy in rheumatoid arthritis. Annals of the rheumatic diseases 69(7): 1275-1280.

33. Beltrametti SP, Ianniello A, Ricci C (2016) Chronotherapy with lowdose modified-release prednisone for the management of rheumatoid arthritis: a review. Ther Clin Risk Manag 12: 1763-1776.

34. Kowanko IC, Pownall R, Knapp MS, Swannell AJ, Mahoney PG (1981) Circadian variations in the signs and symptoms of rheumatoid arthritis and in the therapeutic effectiveness of flurbiprofen at different times of day. Br J Clin Pharmacol 11(5): 477-484.

35. Levi F, Le Louarn C, Reinberg A (1985) Timing optimizes sustainedrelease indomethacin treatment of osteoarthritis. Clinical pharmacology and therapeutics 37(1): 77-84

36. Lotlikar V, Kedar U, Shidhaye S, Kadam V (2010) pH-responsive dual pulse multiparticulate dosage form for treatment of rheumatoid arthritis. Drug Dev Ind Pharm 36(11): 1295-1302.

37. Sunil SA, Srikanth MV, Rao NS, Murthy KV (2013) Chronotherapeutic drug delivery from indomethacin compression coated tablets for early morning pain associated rheumatoid arthritis. Curr Drug Deliv 10(1): 109-121

38. Ramasamy T, Ruttala HB, Shanmugam S, Umadevi SK (2013) Eudragit-coated aceclofenac-loaded pectin microspheres in chrono pharmacological treatment of rheumatoid arthritis. Drug Deliv 20(2): 65-77.

39. Hadi MA, Rao NG, Rao AS (2015) Formulation and evaluation of minitablets-filled-pulsincap delivery of lornoxicam in the chronotherapeutic treatment of rheumatoid arthritis. Pak J Pharm Sci 28(1): 185-193.

40. To H, Irie S, Tomonari M, Watanabe Y, Kitahara T, et al. (2009) Therapeutic index of methotrexate depends on circadian cycling of tumour necrosis factor-alpha in collagen-induced arthritic rats and mice. J Pharm Pharmacol 61(10): 1333-1338

41. To H, Yoshimatsu H, Tomonari M, Ida H, Tsurumoto T, et al. (2011) Methotrexate chronotherapy is effective against rheumatoid arthritis. Chronobiology international 28(3): 267-274.

42. Koyama A, Tanaka A, To H (2017) Daily oral administration of low-dose methotrexate has greater antirheumatic effects in collagen-induced arthritis rats. J Pharm Pharmacol 69(9): 1145-1154.

43. Wang X, Yan X, Wang F, Ge F, Li Z (2018) Role of methotrexate chronotherapy in collagen-induced rheumatoid arthritis in rats. Zeitschrift fur Rheumatologie 77(3): 249-255.

44. Group RC, Horby P, Lim WS, Emberson JR, Mafham M, et al. (2021) Dexamethasone in Hospitalized Patients with Covid-19. The New England journal of medicine 384(8): 693-704. 